\title{
Decreased PD-1 Expression on CD8 Lymphocyte Subsets and Increase in CD8 Tscm Cells in Children with HIV Receiving Raltegravir
}

\author{
Florin Tuluc, ${ }^{1,2}$ Sergei Spitsin,, Nancy B. Tustin,, Jennifer B. Murray,2 Richard Tustin III, \\ Laura A. Schankel, ${ }^{1}$ Andrew Wiznia, ${ }^{3, *}$ Sharon Nachman, ${ }^{4, *}$ and Steven D. Douglas, ${ }^{1,5, *}$
}

\begin{abstract}
We investigated the effect of combination antiretroviral therapy (cART) on immune recovery, particularly on the percentages of PD-1-positive cells within the major leukocyte subsets. Cryopreserved peripheral blood mononuclear cells and plasma samples collected longitudinally from a subset of 13 children and adolescents (between 9.7 and 18.2 years old) who were enrolled in the International Maternal Pediatric Adolescent AIDS Clinical Trials (IMPAACT) P1066 were used for this study. Immunophenotyping by flow cytometry was performed to determine the effect of raltegravir-containing cART regimen on the distribution of leukocyte populations, on the expression of PD-1 on T cell subpopulations, and on the expression of well-established markers of T cell activation (CD38 and HLA-DR) on CD8 T cells. C reactive protein (CRP), lipopolysaccharide (LPS), IL-6, and soluble CD163 were assayed in plasma samples by an enzyme-linked immunosorbent assay. Plasma viral loads were decreased in all subjects (by an average of $2.9 \log$ units). The cART regimen, including raltegravir, induced changes in CD8 T cell subsets, consistent with an effective antiretroviral outcome and improved immunologic status, including increased percentages of CD8 stem cell memory T cells (Tscm). The percentages of CD8 PD-1-positive cells decreased significantly as compared with baseline levels. Among the proinflammatory markers measured in plasma, sCD163 showed a decline that was associated with cART. cART therapy, including raltegravir, over 48 weeks in children is associated with immune restoration, consistent with effective antiretroviral therapy, namely decreased percentages of PD $-1^{+} \mathrm{CD} 8^{+} \mathrm{T}$ cells, an increase in CD8 Tscm cells, and decreased levels of sCD163.
\end{abstract}

Keywords: HIV, antiretroviral therapy, clinical trials for antivirals, integrase inhibitors/drug discovery, $\mathrm{T}$ cell immunity

\section{Introduction}

$\mathbf{T}$ HE DEVELOPMENT OF NOVEL, potent, and well-tolerated antiretroviral (ARV) medications in optimized formulations is a high priority for pediatric patients of all ages who are infected with HIV. Raltegravir is the first integrase inhibitor approved by the Food and Drug Administration and the European Medicines Agency for the treatment of HIV-1 infection based on several clinical studies. ${ }^{1-6}$ Addition of raltegravir to optimized background therapy is well tolerated, with superior and durable antiretroviral and immunologic efficacy compared with optimized background therapy alone.

The International Maternal Pediatric Adolescent AIDS Clinical Trials (IMPAACT) P1066 study was performed to determine the safety and pharmacokinetics of raltegravir in HIV-infected children aged 4 weeks to $<19$ years by using three different formulations. P1066 enrolled participants in 5 age cohorts that received different formulations of raltegravir. ${ }^{7}$ Entry criteria included plasma HIV RNA $>1,000$ copies/mL, previous exposure to ARV but naïve to integrase

\footnotetext{
${ }^{1}$ Division of Allergy and Immunology, The Children's Hospital of Philadelphia Research Institute, Philadelphia, Pennsylvania.

${ }^{2}$ Flow Cytometry Core Laboratory, The Children's Hospital of Philadelphia Research Institute, Philadelphia, Pennsylvania.

${ }^{3}$ Jacobi Medical Center, Albert Einstein College of Medicine, Bronx, New York.

${ }^{4}$ Department of Pediatrics, Stony Brook School of Medicine, Stony Brook, New York.

${ }^{5}$ Department of Pediatrics, Perelman School of Medicine, University of Pennsylvania, Philadelphia, Pennsylvania.

*Members of the International Maternal Pediatric Adolescent AIDS Clinical Trials (IMPAACT) P1066 Protocol Team.
} 
inhibitors, laboratory values below grade 3 toxicity criteria, and absence of opportunistic infections or current cancer. ${ }^{1}$ In addition to providing information on proper dose selection, the P1066 study evaluated the safety, efficacy, and pharmacokinetics properties of raltegravir in children. ${ }^{1}$

The programmed-death 1 (PD-1) protein is inductively expressed on $\mathrm{CD}^{+}, \mathrm{CD}^{+}, \mathrm{NK} \mathrm{T}$ cell subsets, $\mathrm{B}$ cells, and monocytes on activation. PD-1 expression is induced in $\mathrm{T}$ cells by antigen receptor ligation. ${ }^{8}$ PD-1 is transiently expressed in viral-specific CD8 $\mathrm{T}$ cells after infection but once the infection is resolved and T-cell receptor (TCR) signaling diminishes, PD-1 expression decreases. ${ }^{8}$ Conversely, PD-1 expression is maintained during chronic infections, primarily due to continuous TCR ligation. ${ }^{9}$

Upregulation of PD-1 on HIV-specific $\mathrm{CD}^{+}{ }^{+} \mathrm{T}$ cells leads to reversible immune dysfunction, preventing the renewal of the functionally competent HIV-specific $\mathrm{CD}^{+} \mathrm{T}$ cell repertoire. ${ }^{10}$ During chronic HIV infection, virus-specific $\mathrm{CD}^{+} \mathrm{T}$ cells undergo functional exhaustion, lose effector functions, and fail to control viral infection. HIV-specific $\mathrm{CD}^{+} \mathrm{T}$ cells express high levels of co-inhibitory molecule PD-1 during chronic infection and are characterized by decreased proliferation, cytokine production, and cytotoxic abilities. ${ }^{11} \mathrm{PD}-1$ is likewise upregulated on $\mathrm{HIV}$-specific $\mathrm{CD}^{+} \mathrm{T}$ cells, and its expression level correlates with viremia and inversely with $\mathrm{CD}^{+}{ }^{\mathrm{T}}$ cell counts. ${ }^{11}$

Conflicting findings have been reported on the changes in PD-1 levels after combination antiretroviral therapy (cART): Some studies concluded that PD-1 levels on CD4 and CD8 T cells remain high after administering cART to HIV-infected individuals, ${ }^{12}$ whereas others showed evidence that PD-1 expression declines after cART ${ }^{13}$ or is not significantly different in the HIV-1-infected cART-treated group when compared with uninfected controls. ${ }^{14}$

We used cryopreserved peripheral blood mononuclear cells (PBMC) purified from blood samples collected longitudinally (at entry, 8, 24, and 48 weeks) from a subset of participants in the P1066 study. Cryopreservation results in non-linear loss of PD-1 expression that is measured as number of molecules/cell, ${ }^{15,16}$ which complicates the interpretation of findings obtained from retrospective studies. To mitigate this shortcoming, we analyzed the percentage of PD1-positive cells rather than antigen density on target cells.

Our aims were to determine the effects of the raltegravircontaining cART regimen on the percentages of PD-1positive cells within $\mathrm{T}$ cell subpopulations (naïve, central memory, effector memory, terminally differentiated, and stem cell-like memory) and on well-established markers of T cell activation: CD38 and HLA-DR. ${ }^{17-19}$ We also studied the effect of cART on monocyte subsets as defined by the expression of CD14 and CD16 $6^{20,21}$ and on plasma markers of inflammation.

\section{Materials and Methods}

\section{Subjects}

We used cryopreserved blood and plasma samples from a subset of participants in the IMPAACT P1066 study "Safety and Pharmacokinetics of Raltegravir in HIV (Human Immunodeficiency Virus)-Infected Children and Adolescents", (www.clinicaltrials.gov NCT00485264). Subjects were on failing unchanged therapeutic regimen for at least the pre- vious 12 weeks, or treatment experienced (not including therapy to interrupt maternal-infant transmission) but on no treatment for $\geq 4$ weeks before entry. Raltegravir was administered in the background of these failing regimens for up to 14 days and then, the background was optimized for the rest of the study, the details of which have been previously published. ${ }^{1}$

The cells used in this immunophenotyping study were obtained from blood samples collected both before and after treatment with raltegravir at four time points: entry, 8, 24, and 48 weeks. Plasma HIV RNA (RNA) concentrations were determined by using the HIV-1 MONITOR Test, version 1.5 (Roche Molecular Diagnostics) or RealTime HIV-1 (Abbott Molecular). ${ }^{1}$ PBMCs were isolated and cryopreserved, and plasma was frozen and stored at $-80^{\circ} \mathrm{C}$ until it was subsequently analyzed.

We used cryopreserved PBMCs from 12 children aged 12.1-18.2 years who were enrolled in cohort I and one child aged 9.7 years who was enrolled in cohort 2A of the P1066 study. Blood for immunology storage was collected at entry, 8,24 , and 48 weeks. We selected only subjects for whom a sufficient number of PBMC were cryopreserved at all four time points. The availability of sufficient cryopreserved PBMC with cell viability rates $>75 \%$ was the limiting factor that prevented us from extending the study to a larger number of participants. All blood samples used in the present study were collected between May 2008 and November 2010.

Immunophenotyping by multicolor flow cytometry was performed to measure the percentages of PD-1-positive cells within the main subpopulations of $\mathrm{T}$ lymphocytes: naïve (Tn), central memory (Tcm), effector memory (Tem), effector (Te), and stem cell-like memory ( $\mathrm{Tscm}$ ), as previously described. $^{22-24}$

CD38 and HLA-DR were used as markers of $\mathrm{CD}^{+} \mathrm{T}$ cell activation, and percentages of cells expressing either CD38 or both CD38 and HLA-DR were determined. Percentages of $\mathrm{B}$ cells, natural killer cells, and classic (CD14-high CD16 low) and non-conventional (CD14 low, CD16 high) monocytes were also determined.

\section{Flow cytometry}

Multicolor flow cytometry assays were performed by using two panels of antibodies on cryopreserved PBMCs, thawed, and washed in RPMI containing $10 \%$ fetal bovine serum and then in phosphate-buffered saline. Since cells can be damaged by cryopreservation, we used a fixable viability dye to exclude dead cells from analysis. Thawed cells were first stained with the Live/Dead blue viability dye (Life Technologies, Grand Island, NY) to exclude non-viable cells.

For identifying T cell subpopulations and PD-1 expression, PBMCs were then incubated for $30 \mathrm{~min}$ at room temperature in the dark with the following antibodies: CD3-BUV395 (clone UCHT1), CD4-BV650 (OKT4), CD8-BV711 (RPAT8), CD45RO-BV421 (UCHL1), CD197-FITC (G043H7), CD95-APC (DV2), PD-1-PE (EH12.2H7), CD27-PECy7 (O323), HLA-DR-APC-Cy7 (L243), and CD38-PerCPCy5.5 (HB-7). This combination of antibodies was used to measure the percentages of $\mathrm{T}$ cell subsets, mainly based on the expression of CCR7 (CD197), CD45RA, and CD27. The expression of PD-1 on each subset, and the percentages of activated $\mathrm{CD}^{+} \mathrm{T}$ cells $\left(\mathrm{CD} 38^{+}\right.$or $\mathrm{HLA}^{+} \mathrm{DR}^{+}$and $\mathrm{CD} 38^{+}$) 
were also determined. Gating of naïve (Tn), central memory (Tcm), effector memory (Tem), and terminally differentiated effector (Te) T cells was performed as shown in Figure 1 on each of the $\mathrm{CD}^{+}$and $\mathrm{CD} 8^{+} \mathrm{T}$ cell population, following a strategy previously described. ${ }^{24}$

Other lymphocyte subtypes and monocytes were studied on cells stained with CD3-BUV395 (clone UCHT1), CD56BV421 (HCD56), CD19-BV650 (H1B19), CD14-BV711 (M5E2), CD312-FITC (2A1), CD163-PE (MAC2-158), CD16-PE-Cy7 (3G8), CD97-APC (VIM3b), HLA-DR-APCCy7 (L243), and CD38-PerCP-Cy5.5 (HB-7). The identifi- cation of the main populations of leukocytes was performed as previously described. All antibodies were purchased from BioLegend (San Diego, CA), except antibodies for CD163 (Trillium Diagnostics Bangor, ME), CD312 (AbD Serotec, Raleigh, NC), CD97 (eBiosciences, San Diego, CA), and CD3-BUV395 (BD Biosciences, San Jose, CA). Optimal concentrations of antibodies were determined in preliminary titration experiments.

Data acquisition and analyses were performed by using an LSRFortessa flow cytometer and FACSDiva software (BD Biosciences, San Jose, CA). Compensation for fluorescence e PD-1 expression on Tcm, Tem, and $\mathrm{Te} \mathrm{CD}^{+}$cells

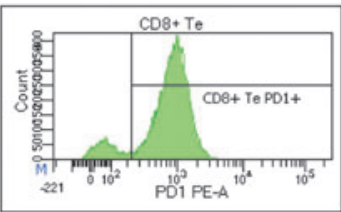

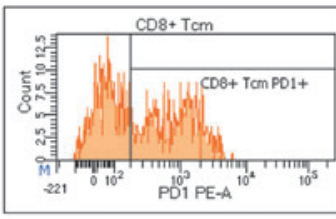

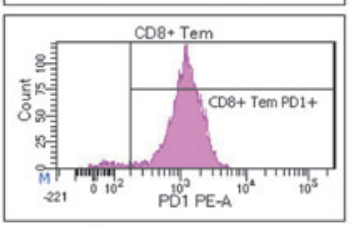

g

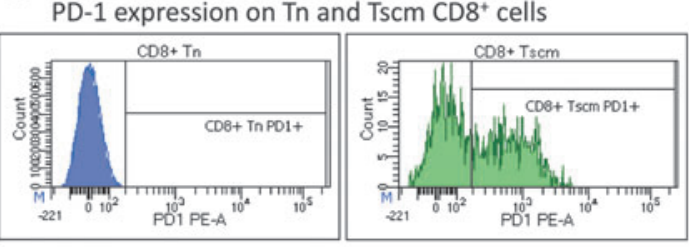

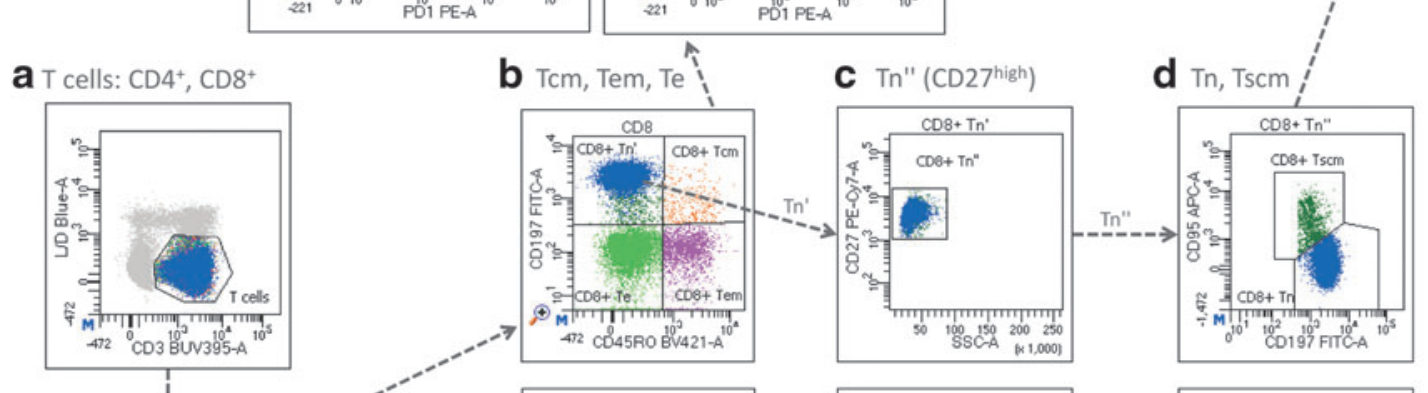
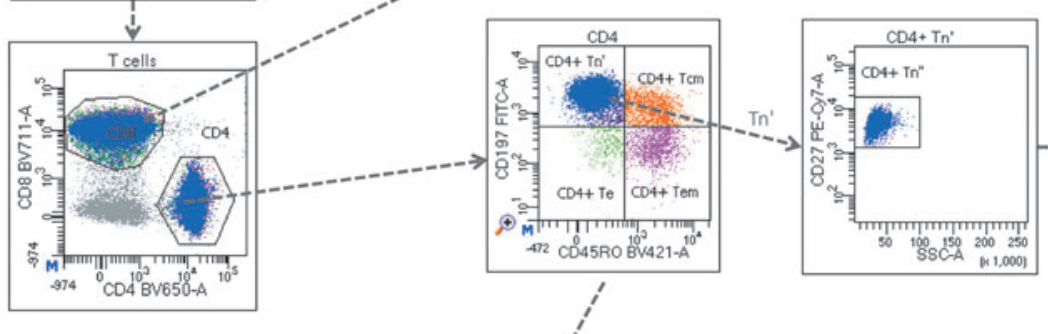

'

f PD-1 expression on Tcm, Tem, and $\mathrm{Te} \mathrm{CD}^{+}$cells
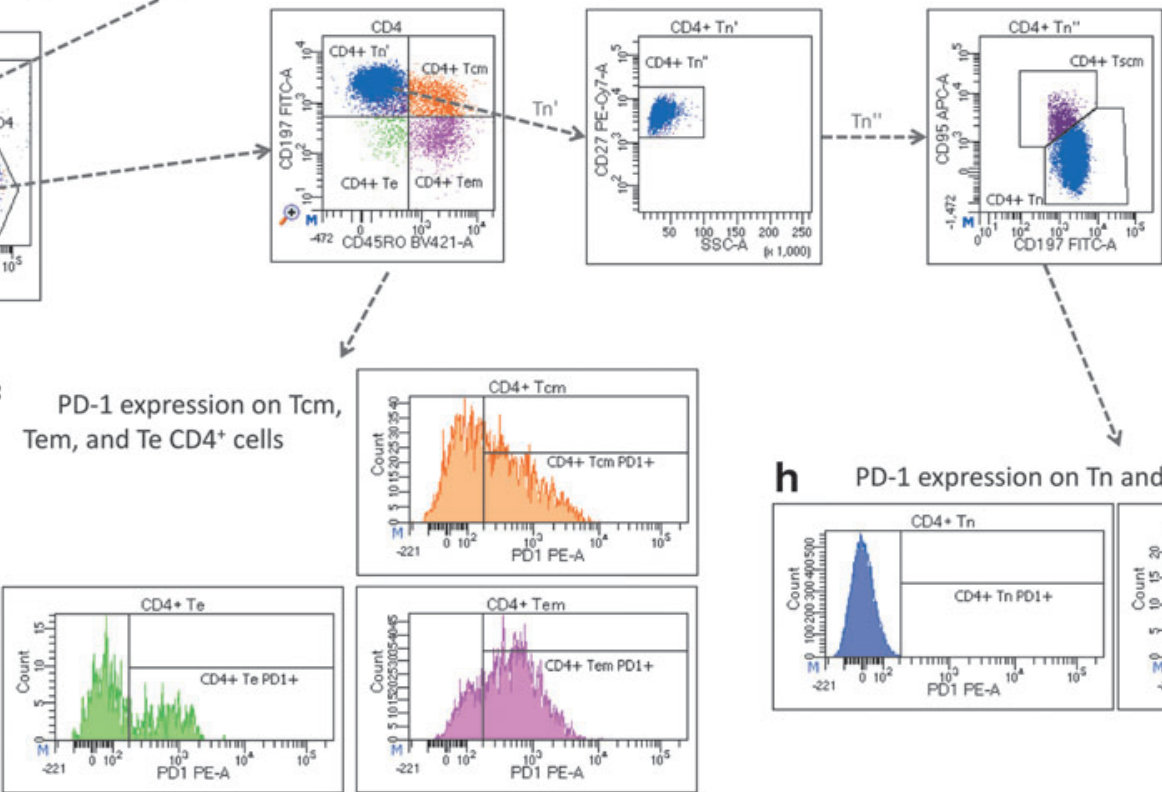

h PD-1 expression on $\mathrm{Tn}$ and $\mathrm{Tscm} \mathrm{CD}^{+}$cells

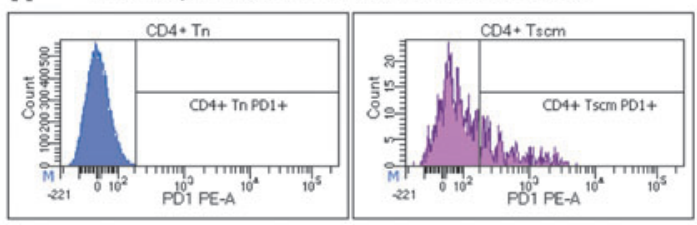

FIG. 1. Gating strategy for identifying T cell subpopulations and the expression pf PD-1. PBMCs were separated from blood samples by centrifugation on Ficoll, and cells were cryopreserved in liquid nitrogen until flow cytometry analysis was performed. Viable $\mathrm{T}$ cells were identified as $\mathrm{CD}^{+}$and $\mathrm{L} / \mathrm{D}$ Blue-dim cells (a), and subpopulations of $\mathrm{CD} 4^{+}$and $\mathrm{CD} 8^{+} \mathrm{T}$ cells were further divided into central memory $(\mathrm{Tcm})$, effector memory (Tem), and effector (Te) $\mathrm{T}$ cells based on the expression of CCR7 (CD197) and CD45RO (b). Naïve and stem cell-like memory T cells (Tscm) were gated based on their high expression of CD27 and low SSC values (c). Tscm cells were distinguished from naïve (Tn) T cells based on higher levels of FasR (CD95) expression (d). Histograms were used for gating PD-1-positive cells in each of the T cell subpopulations described (e-h). PBMC, peripheral blood mononuclear cell. 
spillover was performed for each panel of antibodies in FACSDiva by using OneComp eBeads from eBiosciences (San Diego, CA) stained with each antibody individually and PBMCs stained with Live/Dead blue dye. Gating strategy for identifying the $\mathrm{T}$ cell subsets and the expression of PD-1 is shown in Figure 1.

IL-6 and CRP quantitations were performed by using solid-phase sandwich enzyme-linked immunosorbent assay (ELISA) kits from Invitrogen/Life Technologies (Grand Island, NY).

Soluble CD163 was measured by using an ELISA kit from R\&D Systems (Minneapolis, MN). The procedures provided by the manufacturer were used for each kit, and the absorbance of each well was measured on a BioTek PowerWave XS (BioTek, Winooski, VT).

Lipopolysaccharide (LPS) was measured by using the endpoint chromogenic Limulus Amebocyte Lysate (LAL) test for gram-negative bacterial endotoxin from Lonza (Allendale, $\mathrm{NJ}$ ), following the procedure recommended by the manufacturer. The absorbance of each sample was measured on a Tecan Sunrise ELISA plate reader (Tecan Systems, San Jose, CA).

\section{Statistical analyses}

Two-tailed paired Student's $t$-test was used to test for significant differences, and Bonferroni correction for multiple comparisons on longitudinal samples was applied; 0.01667 was considered the limit for rejecting the null hy- pothesis, accounting for comparisons between values measured at entry versus three subsequent time points $(8,24$, and 48 weeks).

\section{Results}

The cART regimen, including raltegravir, effectively lowered plasma viral loads in all subjects (Fig. 2) from a median value of 23,918 copies/mL at entry to median values of 37,26 , and 14 copies/mL at 8,24 , and 48 weeks, respectively $\left(p<10^{-6}\right.$; Fig. 2a). The range of viral load at entry was between $2.1 \times 10^{3}$ and $2.8 \times 10^{5}$ copies $/ \mathrm{mL}$. After the initiation of cART regimen, all participants had viral loads below 400 copies/mL, except for one subject with viral loads ranging between 424 and 1913 at weeks 24 and 48 .

The percentages of $\mathrm{CD} 4^{+}$cells increased after the initiation of cART treatment containing raltegravir; the mean value at entry was $20.3 \%$ and increased to $22.9 \%$ at 8 weeks, $26.3 \%$ at 24 weeks $(p<.0001)$, and $27.3 \%$ at 48 weeks $(p<.0005$; Fig. 2b). Conversely, the percentages of $\mathrm{CD}^{+} \mathrm{T}$ cells decreased from $53.8 \%$ at entry to $49.1 \%$ at 8 weeks $(p<.0005)$, $45.4 \%$ at 24 weeks $\left(p<10^{-5}\right)$, and $40.6 \%$ at 48 weeks $\left(p<10^{-4}\right.$; Fig. 2c).

We found significant correlations between the percentages of $\mathrm{CD}^{+}$or $\mathrm{CD}^{+} \mathrm{T}$ cells measured in fresh blood samples at the time that the study was conducted with those measured in cryopreserved PBMCs (Fig. 2c, d). Correlations for both CD4 and CD8 were statistically significant $\left(p<10^{-6}\right)$, with Pearson correlation coefficients of 0.89 and 0.75 for CD4\% and
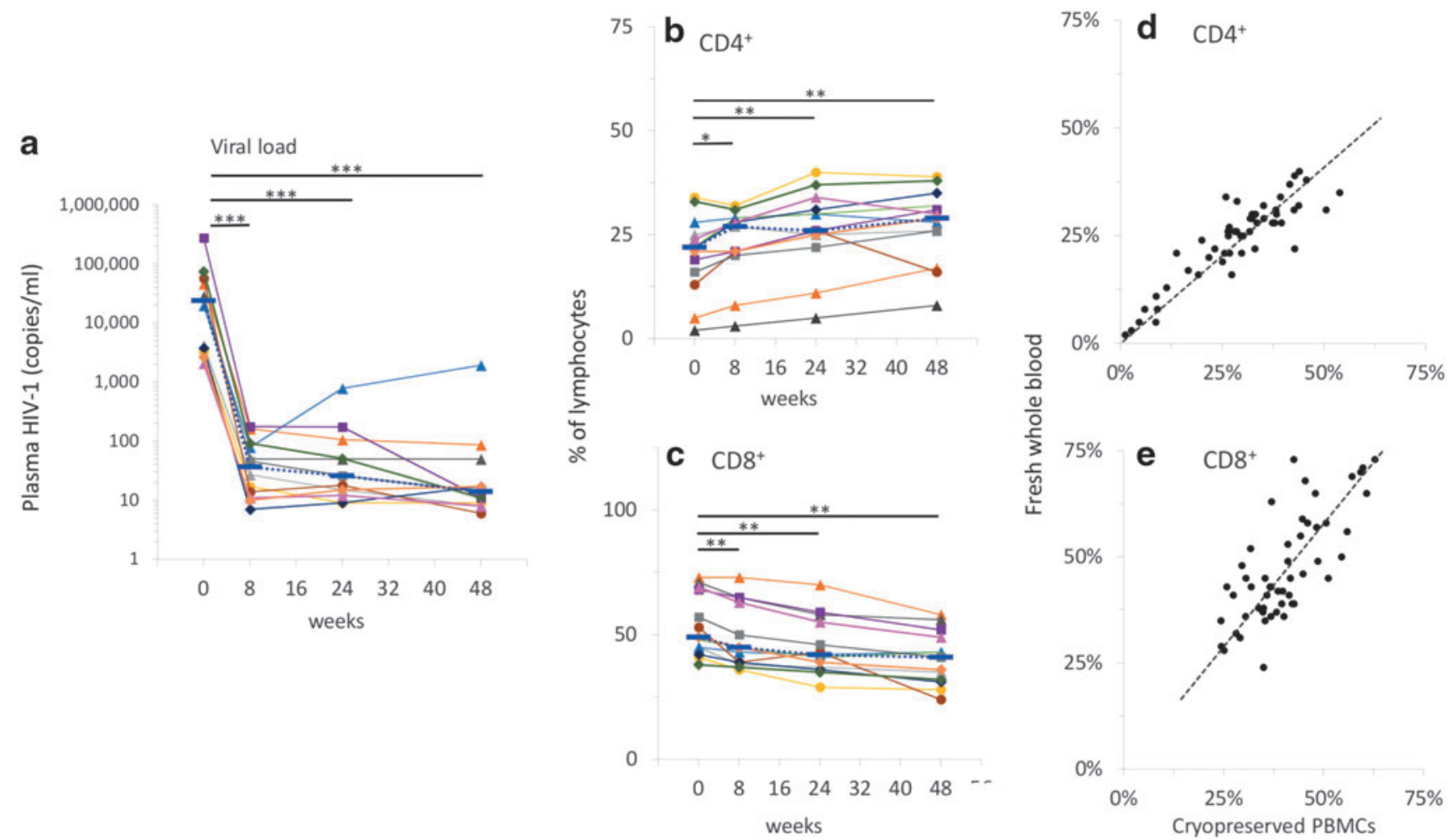

FIG. 2. Viral load and percentages of $\mathrm{CD}^{+}$and $\mathrm{CD}^{+} \mathrm{T}$ cells. Viral loads (a), CD4 (b) and CD8 (c) $\mathrm{T}$ cell percentages in fresh whole blood samples were measured as described under the Materials and Methods section. Individual data points connected by solid lines are shown for each subject; blue dotted lines connect median values. Based on Bonferroni correction for three familywise comparisons, the level of significance for $p$-values was set to .0167. Correlations between $\mathrm{CD}^{+}$(d) and $\mathrm{CD}^{+}$(e) $\mathrm{T}$ cell percentages measured in fresh versus cryopreserved samples were significant for both subpopulations of $\mathrm{CD}^{+}$and $\mathrm{CD}^{+} \mathrm{T}$ cells $\left(p<10^{-6}\right)$. $\left(* p<0.0167 ; * * p<10^{-3} ; * * * p<10^{-6}\right)$. 

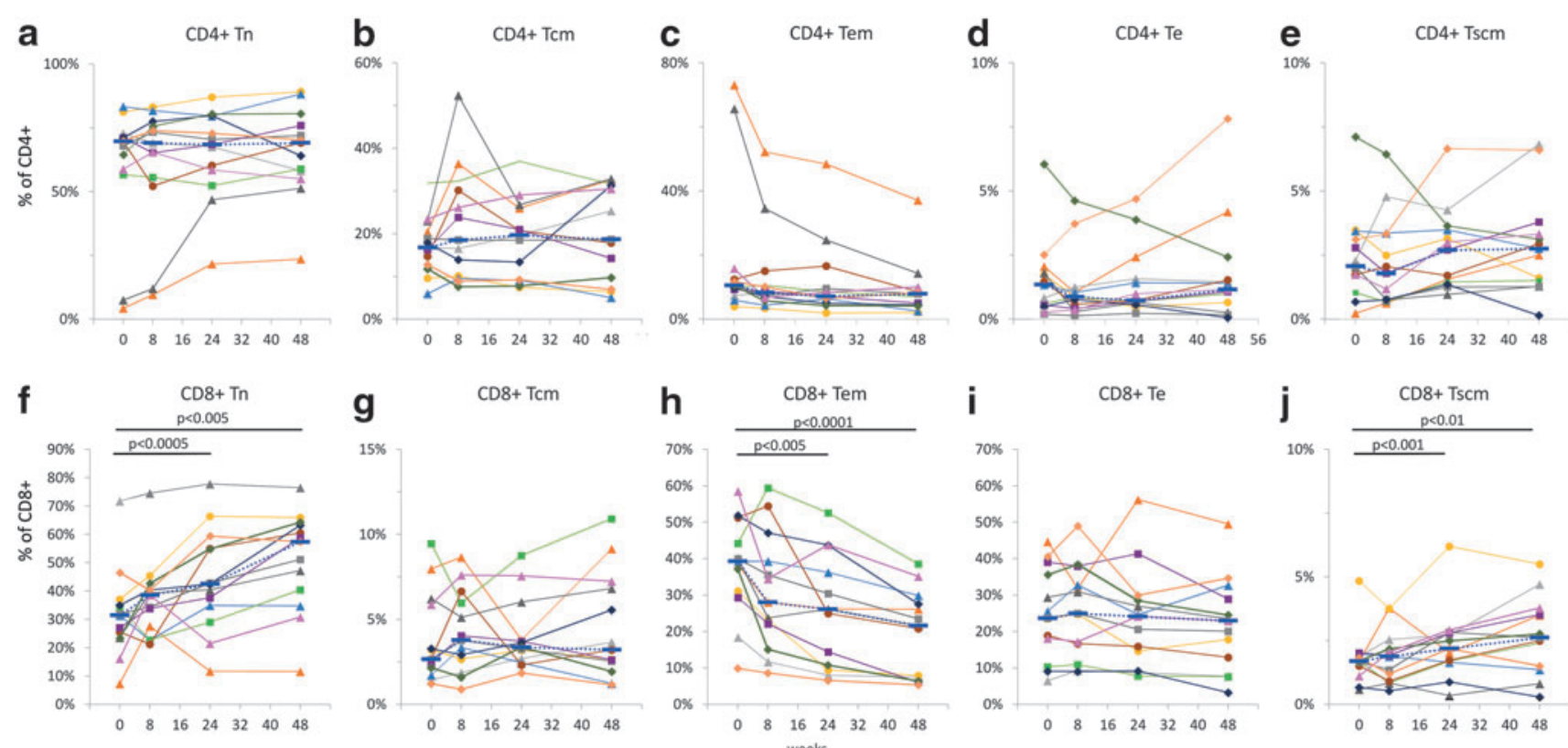

FIG. 3. T cell subpopulations in cryopreserved PBMCs. Percentages of naïve (Tn), central memory (Tcm), effector memory (Tem), effector (Te), and stem cell-like memory (Tscm) cells were determined in CD4 ${ }^{+}(\mathbf{a}-\mathbf{e})$ and CD8+ (f-j) T cells in cryopreserved PBMCs. Individual data points connected by solid lines are shown for each subject; blue dotted lines connect median values. The level of significance for $p$-values was set to .0167 based on Bonferroni correction for three familywise comparisons.

CD8\%, respectively. Differences between median values measured in fresh whole blood samples versus cryopreserved PBMCs were below $4 \%$, consistent with data previously reported. ${ }^{25}$

The percentages of naïve $(\mathrm{Tn})$, central memory $(\mathrm{Tcm})$, effector memory (Tem), effector (Te), and stem cell-like memory (Tscm) cells determined by polychromatic flow cytometry in $\mathrm{CD}^{+}$(Fig. 3a-e) and $\mathrm{CD}^{+}$(Fig. 3f-j) T cells are shown in Figure 3.

The cART regimen containing raltegravir induced significant changes in the distribution of $\mathrm{T}$ cell phenotypes, more prominently in $\mathrm{CD}^{+} \mathrm{T}$ cells. Increased percentages of CD8 Tn were found at 24 and 48 weeks, with median values changing from $31.5 \%$ at entry to $42.5 \%$ at 24 weeks and $57.4 \%$ at 48 weeks, respectively ( $p<.005$; Fig. 3f). A similar trend was observed for $\mathrm{CD}^{+}$Tscm cells, with median percentage values increasing from $1.7 \%$ at entry to $2.2 \%$ and $2.6 \%$ at 24 and 48 weeks, respectively $(p<.01$; Fig. $3 \mathrm{j})$.

Unlike Tn and Tscm cells, median percentage values of CD8 Tem cells decreased from $39.3 \%$ at entry to $26.1 \%$ and $21.6 \%$ at 24 and 48 weeks, respectively ( $p<.001$; Fig. 3 h). The median percentage values at 8 weeks were marginally higher for CD8 Tn (Fig. 3f), and lower for CD8 Tem cells (Fig. 3h) as compared with the corresponding entry values, but the differences were not statistically significant. Neither $\mathrm{CD}^{+} \mathrm{Tcm}$ nor $\mathrm{CD}^{+}$Te cells were changed during the cART regimen (Fig. $3 \mathrm{~g}, \mathrm{i}$ ).

Since the mean fluorescence intensity of PD-1 staining is affected by cryopreservation, ${ }^{15,16}$ we measured the percentage of PD-1-positive cells in each $\mathrm{T}$ cell subpopulation as a primary outcome of the study.

We found that the percentage of total $\mathrm{CD}^{+} \mathrm{PD}^{+}$cells did not change significantly during the study, although the median values trended toward a decrease from $15.6 \%$ at entry to $15.2 \%$ at 8 weeks, $14.5 \%$ at 24 weeks, and $11.1 \%$ at 48 weeks. The percentage of $\mathrm{CD}^{+} \mathrm{PD}-1^{+}$cells decreased significantly from a median value of $44.2 \%$ at entry to $41.0 \%$, $23.5 \%$, and $18.9 \%$ at 8,24 , and 48 weeks, respectively ( $p<.014$; Fig. 4a, g). Percentages of PD-1-positive naïve (Tn), central memory (Tcm), effector memory (Tem), effector (Te), and stem cell-like memory (Tscm) cells were determined for each $\mathrm{CD}^{+}{ }^{+}$(Fig. 4b-f) and $\mathrm{CD}^{+}$(Fig 4h-1) T cell subpopulation in cryopreserved PBMCs. Higher percentages of PD- $1^{+}$CD $4^{+}$Tcm (Fig. $4 c ; p<.01$ ) and CD4 ${ }^{+}$ Tscm (Fig. 4f; $p<.01$ ) cells were measured at 24 and 48 weeks, respectively, whereas the other subpopulations of $\mathrm{CD} 4^{+} \mathrm{T}$ cells did not change significantly during the study.

Among the subpopulations of $\mathrm{CD}^{+} \mathrm{T}$ cells, decreased percentages of cells expressing PD- 1 were found in $\mathrm{CD}^{+} \mathrm{Tcm}$ (48 weeks; Fig. 4i) and Te (24 and 48 weeks; Fig. 4j) cells.

We measured significant changes in activation markers CD38 and HLA-DR on $\mathrm{CD}^{+} \mathrm{T}$ cells at all time points after the initiation of cART. Percentages of $\mathrm{CD} 38^{+} \mathrm{CD} 8^{+}-\mathrm{T}$ cells or dual positive for $\mathrm{HLA}-\mathrm{DR}^{+}$and $\mathrm{CD}^{+} 8^{+}$significantly decreased at 8 weeks and remained lower than at the beginning of the study (Fig. 5). Viral loads were significantly correlated with $\mathrm{CD}^{+} \mathrm{CD}^{+} 8^{+}$(Fig 5c) and $\mathrm{CD}^{+} \mathrm{CD}^{+} 8^{+} \mathrm{HLA}_{-} \mathrm{DR}^{+}$ (Fig. 5d); Pearson coefficients were 0.58 and 0.70 , respectively $\left(p<10^{-5}\right)$.

No significant differences were found between percentages of B cells, monocytes, NK cells, or total T cells measured at entry as compared with the other time points in the study (data not shown).

We measured concentrations of CRP, LPS, IL-6, and soluble CD163 in cryopreserved plasma samples from the same study participants (Fig. 5e-h). No significant differences were detected between the levels of CRP, LPS, and IL6. 


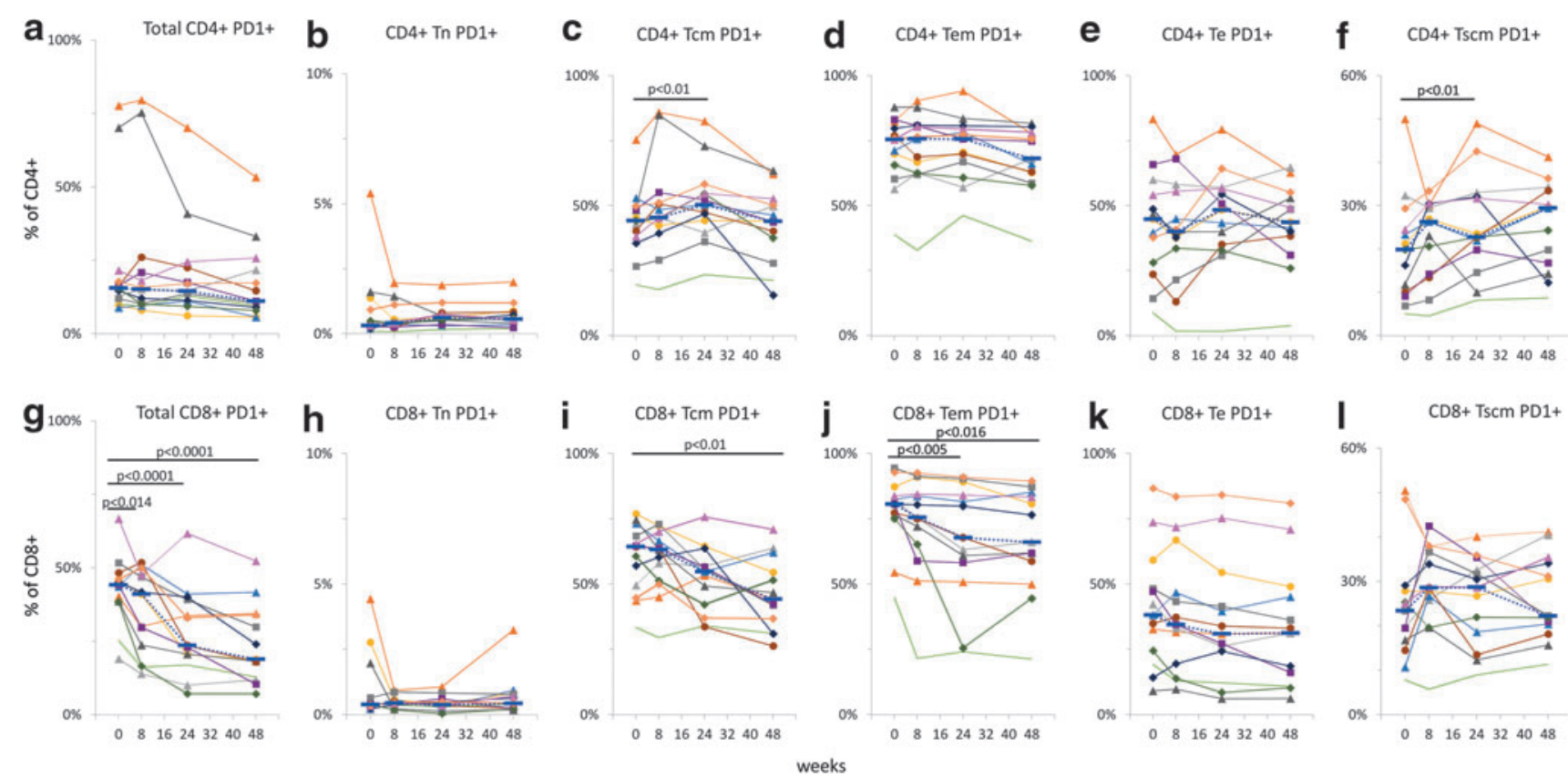

FIG. 4. Expression of PD-1 on T cells. Percentages of PD-1-positive T cells, total, naïve (Tn), central memory (Tcm), effector memory (Tem), effector (Te), and stem cell-like memory (Tscm) cells were determined in cryopreserved PBMCs for $\mathrm{CD}^{+}(\mathbf{a}-\mathbf{f})$ and $\mathrm{CD}^{+}(\mathbf{g}-\mathbf{l}) \mathrm{T}$ cells. Individual data points connected by solid lines are shown for each subject; blue dotted lines connect median values. Based on Bonferroni correction for three familywise comparisons, the level of significance for $p$-values was set to .0167.

a
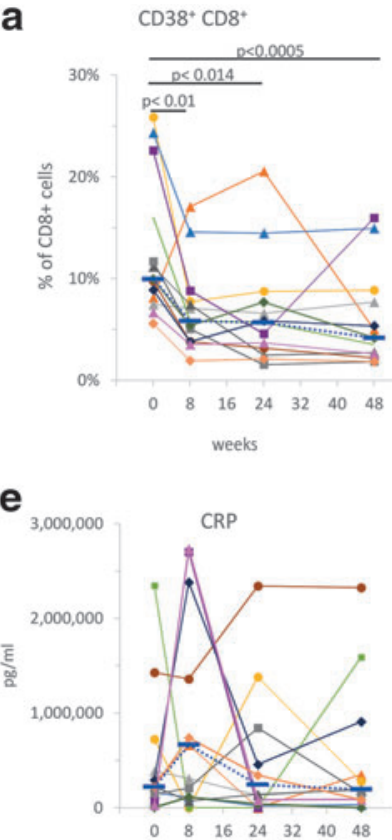

b $\quad \mathrm{CD} 38^{+} \mathrm{HLA}-\mathrm{DR}^{+} \mathrm{CD} 8^{+}$

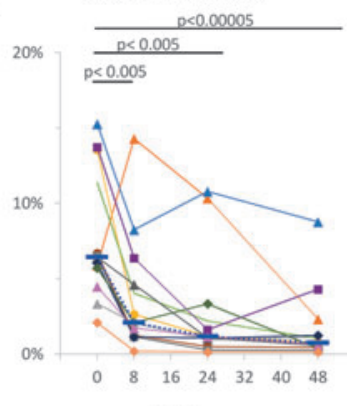

weeks

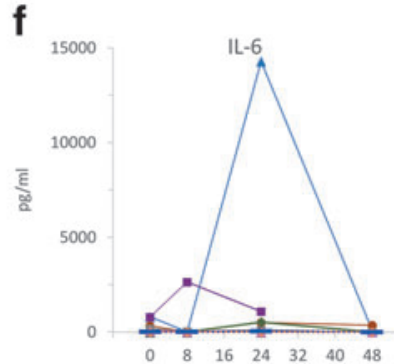

C $\mathrm{CD}_{3} 8^{+} \mathrm{CD} 8^{+}$

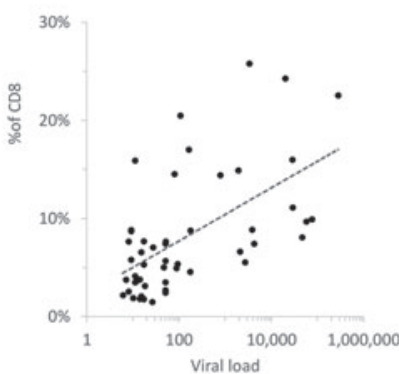

g

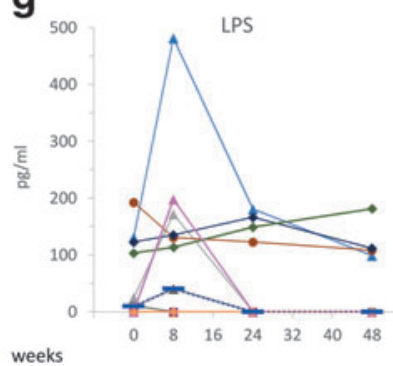

d $\quad \mathrm{CD}^{*} 8^{+} \mathrm{HLA}-\mathrm{DR} \mathrm{CD}^{*}$

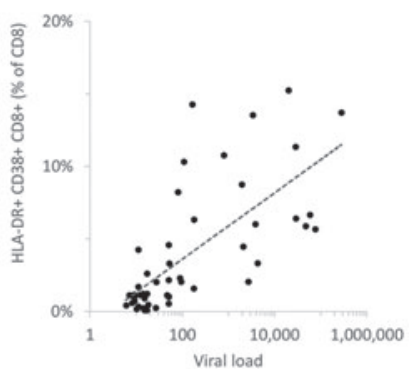

h

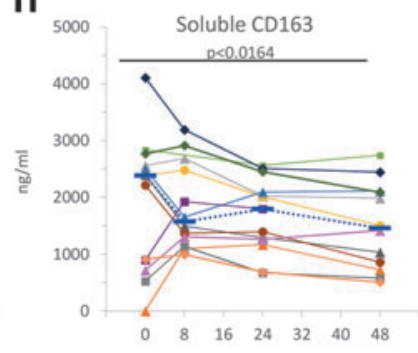

FIG. 5. Markers of immune activation on CD8 T cells and inflammation markers in plasma. After the initiation of cART, the percentages of $\mathrm{CD}^{+}$-T cells positive for CD38 or dual positive for HLA-DR and CD38 significantly decreased at 8 weeks and remained lower than at the beginning of the study $(\mathbf{a}, \mathbf{b})$. Correlations between viral loads and CD $8^{+} \mathrm{CD} 38^{+}(\mathbf{c})$ or $\mathrm{CD}^{+} \mathrm{CD} 8^{+} \mathrm{HLA}^{-\mathrm{DR}^{+}}$(d) are shown. Individual data points connected by solid lines are shown for each patient; dotted lines in (a) and (b) connect median values. Plasma levels of CRP (e), IL-6 (f), LPS (g), and soluble CD163 (h) were measured as described under the Materials and Methods section. Individual data points connected by solid lines are shown for each subject; dotted lines connect median values. The level of significance for $p$-values was set to .0167. cART, combination antiretroviral therapy; CRP, C reactive protein; LPS, lipopolysaccharide. 
Mean plasma concentrations of sCD163 significantly decreased from $2.1 \mu \mathrm{g} / \mathrm{mL}$ at entry to $1.5 \mu \mathrm{g} / \mathrm{mL}$ at week 48 $(p<.0164$; Fig. 5h).

\section{Discussion}

Intensification of cART regimen with raltegravir in children and adolescents failing a therapeutic regimen effectively decreased viral loads and increased the percentage of $\mathrm{CD}^{+}$ cells. We demonstrated that effective antiviral therapy is associated with a decrease in PD- 1 expression on $\mathrm{CD}^{+}$but not $\mathrm{CD} 4^{+}$cells, an increase in percentage of $\mathrm{CD} 8^{+} \mathrm{Tscm}$, and a decrease in markers of immune activation (CD38 and HLADR). This is the first report of the effect of antiviral therapy on PD-1 expression by lymphocytes in children and adolescents. By markedly reducing HIV replication, cART eventually triggers an often slow and incomplete recovery of the immune system toward normality. ${ }^{26}$ In adults, effective cART leads to a significant decline in viremia and an increase in percentages of CD4 T cells within 1 month from the beginning of therapy, mainly due to increased percentages of central memory $\mathrm{CD}^{+}{ }^{+} \mathrm{T}$ cells. ${ }^{27-29}$ After several months of treatment, naïve $\mathrm{CD} 4^{+}$lymphocytes also increase whereas $\mathrm{CD}^{+} \mathrm{T}$ cells decline.

Unlike adults, children respond with a preferential expansion of the naïve $T$ cell pool after initiating successful cART, probably due to the presence of a functioning thymus. ${ }^{30}$ HIV infection may not uniformly result in accelerated thymic involution in childhood, but studies based on the assessment of $\mathrm{T}$ cell receptor rearrangement excision circles demonstrated that thymopoiesis can occur in adolescents who are perinatally infected, despite lifelong infection. ${ }^{31}$ The percentage of $\mathrm{CD}^{+}$cells recently emigrated from thymus predicts disease progression and may reflect history of disease in HIV-positive adults and adolescents. ${ }^{32}$ Many individuals after up to two decades of infection controlled with antiretroviral therapy have sufficient thymic reserve to compensate for CD4 T-cell loss. ${ }^{33}$ Inter-individual responses to cART vary considerably ${ }^{34}$ and HIV-specific $\mathrm{CD}^{+}{ }^{+} \mathrm{T}$ cell responses are rarely recovered, ${ }^{35}$ with normalization of the $\mathrm{CD} 4^{+} /$ $\mathrm{CD}^{+} \mathrm{T}$ cell ratio occurring in only a minority of cases. ${ }^{36}$

Viral loads as well as percentages and absolute counts of $\mathrm{CD}^{+}$and $\mathrm{CD}^{+} \mathrm{T}$ cells for all subjects enrolled in the P1066 have already been published (Nachman et al., 2013). ${ }^{1}$ The selection of participants depended on the availability of at least four time points of cryopreserved PBMC samples with cell viability rates above $75 \%$.

Consistent with previous studies and guidelines of quality assurance, ${ }^{25,37,38}$ we found significant correlations between the percentages of CD4 and CD8 T cells measured in fresh samples versus cryopreserved samples, confirming that the cell surface markers were intact. Nevertheless, it should be noted that the CD4 and CD8 assays in fresh blood were performed in different laboratories, and inter-laboratory variability is expected for these type of assays. Furthermore, the PBMC isolation procedure may also be a source of variability.

The distribution of CD4 $\mathrm{T}$ cell subtypes did not change significantly. However, two of the subjects had low percentages of $\mathrm{CD} 4 \mathrm{~T}$ cells ( $2 \%$ and $5 \%$ ), low CD4 naïve (4.2\% and $7.4 \%$ respectively), and corresponding high percentages of CD4 effector memory $\mathrm{T}$ cells $(73.1 \%$ and $65.7 \%$, respec- tively) at entry. One of these two patients had multiple infections (pneumonia, recurrent varicella, sinusitis, and oral candidiasis) and presented generalized and persistent lymphadenopathy; the other subject with low CD4 counts had oral candidiasis but no other infections. Both patients were men and showed improved immunologic status during the regimen with raltegravir. The other 11 subjects had CD4 Tn cells above $50 \%$ throughout the study. Taken together, this group of subjects did not show significant changes in CD4 T cell distribution. This finding may be the consequence of previous cART treatment and the very brief 3 weeks of interruption of cART before starting the raltegravir regimen.

Tscm CD4 cells are potential cellular targets for reducing HIV reservoirs, ${ }^{39}$ and high levels of PD-1 expression on CD4 cells predict suboptimal CD4 $\mathrm{T}$ cell recovery after long-term cART. $^{12}$ The PD-1 expression on CD4 T cells subsets did not decrease over time, possibly due to the fact that the subjects were previously under cART.

We found a significant increase of CD8 Tn and Tscm CD8 T cells at 24 and 48 weeks as compared with baseline and percentages of CD8 Tem cells decreased significantly at 24 and 48 weeks, consistent with decreased immune activation induced by effective cART therapy.

The frequency of CD8 Tscm has been reported to decrease in all individuals with chronic, untreated HIV-1 infection. cART has a restorative effect on this subset, and high levels of circulating Tscm cells are associated with improved prognosis in chronic HIV-1 infection. ${ }^{40}$ Our data provide further evidence that CD8 Tscm cells increase with cART, and this finding may be used as an indicator of effective cART therapy.

PD-1 has been investigated for its possible roles in HIV pathogenesis. ${ }^{41}$ Cryopreservation has an impact on the level of expression of PD-1. ${ }^{15,16}$ However, due to a limited availability of fresh human PBMC, cryopreserved cells were used to study PD-1 expression. ${ }^{13,41}$ In general, mean fluorescence intensity correlates with the frequency of $\mathrm{PD}-1^{+}$cells. ${ }^{13}$ Based on our experience of the greater effect of cryopreservation on mean fluorescence intensity of PD-1 staining, ${ }^{15,16}$ we focused on measuring the percentages of PD-1-positive cells. The percentage of Tcm CD4 cells that expressed PD-1 was higher at 24 weeks than at baseline, but this change did not persist at 48 weeks. An increase of Tscm CD4 cells that expressed PD-1 was detected for the Tscm cells at 24 weeks, but the differences were not significant at 8 or 48 weeks.

Effective cART along with immune restoration is associated with a decrease in PD-1 expression by CD4 T cells. ${ }^{42,43}$ We detected a moderate increase in the percentage of PD-1 expressing CD4-positive $\mathrm{T}$ cells during the first 2 months of cART therapy in an adult cohort. ${ }^{44}$ The transient increase in PD-1 levels may be due to incomplete viral suppression, leading to continued exposure of the immune system to low levels of viral antigens or/and to early $\mathrm{T}$ cell reconstitution from a lymphoid tissue where PD-1 expression may be higher. ${ }^{43}$

The most important changes in PD-1 expression were detected for the CD8 T cells. Percentages of total PD-1positive cells were lower than at baseline, due mainly to decreased percentages of Tcm and Tem cells. Tcm displayed significantly lower levels of PD-1 expression at 48 weeks only, whereas Tem showed a significant decrease at both 24 and 48 weeks. Breton et ll $^{41}$ showed higher expression 
levels for PD-1 measured as mean fluorescence intensity on all $\mathrm{T}$ cell subsets in chronic HIV-infected subjects without treatment versus subjects on cART. We found a decrease of PD-1 expression (measured as percentage of total PD-1positive cells) in $\mathrm{CD}^{+} \mathrm{T}$ cells after the initiation of $\mathrm{cART}$ (Fig. 4g). However, the percentages of PD-1-positive CD4 T cells did not change significantly after cART (Fig. 4a-f). The percentages of activated CD8 T cells that express CD38 and/ or HLA-DR were the most rapid changes that signaled effective therapy (Fig. 5a, b).

We also measured the percentages of NK cells, B cells, and monocyte subsets but we did not find significant changes over time. Although we anticipated to find decreased percentages in $\mathrm{CD}_{16} 6^{+} \mathrm{CD} 14^{\text {low }}$ non-conventional monocytes after the initiation of effective cART, we did not observe such a decrease, possibly due to small sample size.

An increase in the plasma levels of proinflammatory markers is associated with HIV infection, whereas the suppression of HIV replication with antiviral therapy leads to at least partial resolution of chronic inflammation. ${ }^{45-49}$ Among plasma levels of several proinflammatory markers measured, CRP, IL6, sCD163, and LPS, only sCD163 was significantly decreased by week 48 , consistent with other reports. ${ }^{18}$ Lack of changes in CRP levels despite viral suppression was also previously reported. ${ }^{18}$ Long-term cART has been associated with a decrease in plasma LPS levels in adults. ${ }^{50} \mathrm{We}$ observed no change in the plasma LPS level in this study of children. These may be due to patient age and study duration.

Our data indicate that raltegravir improves immune status, consistent with effective antiretroviral therapy. Decreased expression of PD-1, CD38, and HLA-DR on CD8 ${ }^{+}$T-cells, an increase in CD8 Tscm cells, and decreased levels of sCD163 are markers of immune restoration in response to cART with raltegravir intensification.

\section{Acknowledgments}

This work was supported by U.S. National Institutes of Health (NIH) grants P01 MH105303 (to SDD), R21 AI 108296 (to SDD), Penn Mental Health AIDS Research Center (P30 MH097488), and Penn Center for AIDS Research (P30 AI045008).

Overall support for the IMPAACT Group was provided by the National Institute of Allergy and Infectious Diseases (NIAID) of the NIH under award numbers UM1AI068632 (IMPAACT LOC), UM1AI068616 (IMPAACT SDMC), and UM1AI106716 (IMPAACT LC), with co-funding from the Eunice Kennedy Shriver National Institute of Child Health and Human Development (NICHD) and the National Institute of Mental Health (NIMH). The content is solely the responsibility of the authors and does not necessarily represent the official views of the NIH.

Members of the P1066 Protocol Team include: Nan Zheng MA, Terence Fenton, Ed D, and Carmelito Alvero, MS, Statistical and Data Analysis Center, Harvard School Public Health, Boston, MA; Edward Acosta, Pharm D, University of Alabama at Birmingham, Birmingham, AL; Hedy Teppler, MD, Brenda Homony, MS, Xia Xu, PhD, Larissa Wenning, $\mathrm{PhD}$, Matthew Rizk, PhD, Merck\&Co.,West Point, PA; Carrie Fry, BS and Bobbie Graham, BS, Frontier Science and Technology Research Foundation, Amherst, NY; Stephen A. Spector, MD, University of California, San Diego and Rady
Children's Hospital San Diego, La Jolla, CA; Lisa M. Frenkel, MD, University of Washington Children's Hospital, Seattle, WA; Carol Worrell, MD, Pediatric Adolescent and Maternal AIDS Branch, Eunice Kennedy Shriver National Institute of Child Health and Development, Bethesda, MD; Ed Handelsman, MD, Paul Sato, MD, Division of AIDS, National Institute of Allergy and Infectious Diseases, Bethesda, MD.

\section{Author Disclosure Statement}

No competing financial interests exist.

\section{References}

1. Nachman S, Zheng N, Acosta EP, et al.: Pharmacokinetics, safety, and 48-week efficacy of oral raltegravir in HIV-1infected children aged 2 through 18 years. Clin Infect Dis 2013;58:413-422.

2. Lennox JL, DeJesus E, Lazzarin A, et al.: Safety and efficacy of raltegravir-based versus efavirenz-based combination therapy in treatment-naive patients with HIV-1 infection: A multicentre, double-blind randomised controlled trial. Lancet (London, England) 2009;374:796-806.

3. Rockstroh JK, DeJesus E, Lennox JL, et al.: Durable efficacy and safety of raltegravir versus efavirenz when combined with tenofovir/emtricitabine in treatment-naive HIV-1infected patients: Final 5-year results from STARTMRK. J Acquir Immune Defic Syndr 2013;63:77-85.

4. De Castro N, Braun J, Charreau I, et al.: Switch from enfuvirtide to raltegravir in virologically suppressed multidrug-resistant HIV-1-infected patients: A randomized open-label trial. Clin Infect Dis 2009;49:1259-1267.

5. Steigbigel RT, Cooper DA, Kumar PN, et al.: Raltegravir with optimized background therapy for resistant HIV-1 infection. N Engl J Med 2008;359:339-354.

6. Steigbigel RT, Cooper DA, Teppler H, et al.: Long-term efficacy and safety of Raltegravir combined with optimized background therapy in treatment-experienced patients with drug-resistant HIV infection: week 96 results of the BENCHMRK 1 and 2 Phase III trials. Clin Infect Dis 2010; 50:605-612.

7. Rizk ML, Du L, Bennetto-Hood C, et al.: Population pharmacokinetic analysis of raltegravir pediatric formulations in HIV-infected children 4 weeks to 18 years of age. J Clin Pharmacol 2015;55:748-756.

8. Kamphorst AO, Ahmed R: Manipulating the PD-1 pathway to improve immunity. Curr Opin Immunol 2013;25:381-388.

9. Blattman JN, Wherry EJ, Ha S-J, van der Most RG, Ahmed $\mathrm{R}$ : Impact of epitope escape on PD-1 expression and CD8 T-cell exhaustion during chronic infection. J Virol 2009;83:4386-4394.

10. Trautmann L, Janbazian L, Chomont N, et al.: Upregulation of PD-1 expression on HIV-specific CD8+ T cells leads to reversible immune dysfunction. Nat Med 2006;12: 1198-1202.

11. Day CL, Kaufmann DE, Kiepiela P, et al.: PD-1 expression on HIV-specific T cells is associated with T-cell exhaustion and disease progression. Nature 2006;443:350-354.

12. Kulpa DA, Lawani M, Cooper A, Peretz Y, Ahlers J, Sékaly R-P: PD-1 coinhibitory signals: The link between pathogenesis and protection. Semin Immunol 2013;25:219227.

13. Cockerham LR, Jain V, Sinclair E, et al: : Programmed death- 1 expression on $\mathrm{CD}^{+}$and $\mathrm{CD}^{+} \mathrm{T}$ cells in treated and untreated HIV disease. AIDS 2014;28: 1749-1758. 
14. Rosignoli G, Cranage A, Burton C, et al.: Expression of PD-L1, a marker of disease status, is not reduced by HAART in aviraemic patients. AIDS 2007;21:1379-1381.

15. Holm M, Pettersen FO, Kvale D: PD-1 predicts CD4 loss rate in chronic HIV-1 infection better than HIV RNA and CD38 but not in cryopreserved samples. Curr HIV Res 2008;6:49-58.

16. Campbell DE, Tustin NB, Riedel E, et al.: Cryopreservation decreases receptor PD-1 and ligand PD-L1 coinhibitory expression on peripheral blood mononuclear cellderived $\mathrm{T}$ cells and monocytes. Clin Vaccine Immunol 2009;16:1648-1653.

17. Flynn PM, Rudy BJ, Douglas SD, et al.: Virologic and immunologic outcomes after 24 weeks in HIV type 1infected adolescents receiving highly active antiretroviral therapy. J Infect Dis 2004;190:271-279.

18. Rudy BJ, Kapogiannis BG, Worrell C, et al.: Immune reconstitution but persistent activation after 48 weeks of antiretroviral therapy in youth with pre-therapy CD4>350 in ATN 061. J Acquir Immune Defic Syndr 2015;69:52-60.

19. Starr SE, Sarr M, Campbell DE, Wilson CM, Douglas SD: Increased proliferation within $\mathrm{T}$ lymphocyte subsets of HIV-infected adolescents. AIDS Res Hum Retroviruses 2002; 18:1301-1310.

20. Geissmann F, Jung S, Littman DR: Blood monocytes consist of two principal subsets with distinct migratory properties. Immunity 2003;19:71-82.

21. Ziegler-Heitbrock L, Ancuta P, Crowe S, et al.: Nomenclature of monocytes and dendritic cells in blood. Blood 2010;116:e74-e80.

22. Maecker HT, McCoy JP, Nussenblatt R: Standardizing immunophenotyping for the human immunology project. Nat Rev Immunol 2012;12:191-200.

23. Gattinoni L, Lugli E, Ji Y, et al:: A human memory T cell subset with stem cell-like properties. Nat Med 2011;17: 1290-1297.

24. Lugli E, Gattinoni L, Roberto A, et al.: Identification, isolation and in vitro expansion of human and nonhuman primate T stem cell memory cells. Nat Protoc 2013;8: 33-42.

25. Weinberg A, Song L-Y, Wilkening C, et al.: Optimization and limitations of use of cryopreserved peripheral blood mononuclear cells for functional and phenotypic T-cell characterization. Clin Vaccine Immunol 2009;16:11761186.

26. Douek DC, Roederer M, Koup RA: Emerging concepts in the immunopathogenesis of AIDS. Annu Rev Med 2009; 60:471-484.

27. Muñoz-Calleja C, Costantini A, Silvestri G, et al.: Highly active antiretroviral therapy induces specific changes in effector and central memory $\mathrm{T}$ cell sub-populations. AIDS 2001;15:1887-1890.

28. Autran B, Carcelain G, Li TS, et al.: Positive effects of combined antiretroviral therapy on CD4+ T cell homeostasis and function in advanced HIV disease. Science 1997; 277:112-116.

29. Pakker NG, Notermans DW, de Boer RJ, et al.: Biphasic kinetics of peripheral blood $\mathrm{T}$ cells after triple combination therapy in HIV-1 infection: A composite of redistribution and proliferation. Nat Med 1998;4:208-214.

30. Gibb DM, Newberry A, Klein N, de Rossi A, GroschWoerner I, Babiker A: Immune repopulation after HAART in previously untreated HIV-1-infected children. Paediatric
European Network for Treatment of AIDS (PENTA) Steering Committee. Lancet (London, England) 2000;355: 1331-1332.

31. Pham T, Belzer M, Church JA, et al.: Assessment of thymic activity in human immunodeficiency virus-negative and positive adolescents by real-time PCR quantitation of Tcell receptor rearrangement excision circles. Clin Diagn Lab Immunol 2003;10:323-328.

32. Zakhour R, Tran DQ, Degaffe G, et al.: Recent thymus emigrant CD4+ T cells predict HIV disease progression in patients with perinatally acquired HIV. Clin Infect Dis 2016;62:1029-1035.

33. Aguilera-Sandoval CR, Yang OO, Jojic N, et al.: Supranormal thymic output up to 2 decades after HIV-1 infection. AIDS 2016;30:701-711.

34. Aiuti F, Mezzaroma I: Failure to reconstitute CD4+ T-cells despite suppression of HIV replication under HAART. AIDS Rev 2006;8:88-97.

35. Pitcher CJ, Quittner C, Peterson DM, et al.: HIV-1-specific CD4+ $\mathrm{T}$ cells are detectable in most individuals with active HIV-1 infection, but decline with prolonged viral suppression. Nat Med 1999;5:518-525.

36. Leung V, Gillis J, Raboud J, et al.: Predictors of CD4:CD8 ratio normalization and its effect on health outcomes in the era of combination antiretroviral therapy. PLoS One 2013; 8:e77665.

37. Bull M, Lee D, Stucky J, et al.: Defining blood processing parameters for optimal detection of cryopreserved antigenspecific responses for HIV vaccine trials. J Immunol Methods 2007;322:57-69.

38. Sarzotti-Kelsoe M, Needham LK, Rountree W, et al.: The Center for HIV/AIDS Vaccine Immunology (CHAVI) multi-site quality assurance program for cryopreserved human peripheral blood mononuclear cells. J Immunol Methods 2014;409:21-30.

39. Chahroudi A, Silvestri G, Lichterfeld M: T memory stem cells and HIV: A long-term relationship. Curr HIV/AIDS Rep 2015;12:33-40.

40. Ribeiro SP, Milush JM, Cunha-Neto E, et al:: The CD8 ${ }^{+}$ memory stem $\mathrm{T}$ cell (T(SCM)) subset is associated with improved prognosis in chronic HIV-1 infection. J Virol 2014;88:13836-13844.

41. Breton G, Chomont N, Takata H, et al.: Programmed death1 is a marker for abnormal distribution of naive/memory $\mathrm{T}$ cell subsets in HIV-1 infection. J Immunol 2013;191:21942204.

42. Porichis F, Kaufmann DE: Role of PD-1 in HIV pathogenesis and as target for therapy. 2012;9:81-90.

43. D'Souza M, Fontenot AP, Mack DG, et al.: Programmed death 1 expression on HIV-specific CD4+ T cells is driven by viral replication and associated with $\mathrm{T}$ cell dysfunction. J Immunol 2007;179:1979-1987.

44. Spitsin S, Tustin NB, Riedel E, et al.: Programmed death 1 receptor changes Ex Vivo in HIV-infected adults following initiation of highly active antiretroviral therapy. Clin Vaccine Immunol 2012;19:752-756.

45. Ipp H, Zemlin AE, Erasmus RT, Glashoff RH: Role of inflammation in HIV-1 disease progression and prognosis. Crit Rev Clin Lab Sci 2014;51:98-111.

46. French MA, King MS, Tschampa JM, da Silva BA, Landay AL: Serum immune activation markers are persistently increased in patients with HIV infection after 6 years of antiretroviral therapy despite suppression of viral replication 
and reconstitution of CD4+ T cells. J Infect Dis 2009; 200:1212-1215.

47. Rajasuriar R, Khoury G, Kamarulzaman A, French MA, Cameron PU, Lewin SR: Persistent immune activation in chronic HIV infection: Do any interventions work? AIDS 2013;27:1199-1208.

48. Stein JH, Hsue PY: Inflammation, immune activation, and CVD risk in individuals with HIV infection. JAMA 2012; 308:405-406.

49. French MA, Cozzi-Lepri A, Arduino RC, Johnson M, Achhra AC, Landay A: Plasma levels of cytokines and chemokines and the risk of mortality in HIV-infected individuals: A case-control analysis nested in a large clinical trial. AIDS 2015;29:847-851.

50. Vassallo M, Mercié P, Cottalorda J, Ticchioni M, Dellamonica P: The role of lipopolysaccharide as a marker of immune activation in HIV-1 infected patients: A systematic literature review. Virol J 2012;9:174.

Address correspondence to: Steven D. Douglas Department of Pediatrics Perelman School of Medicine University of Pennsylvania Children's Hospital of Philadelphia 34th Street and Civic Center Boulevard Abramson Building Room 1208A Philadelphia, PA 19104

E-mail: douglas@email.chop.edu 